\title{
Exercise-Induced Inflammatory Response: To Use or Not use Anti-Inflammatory Medication
}

\author{
Albená Nunes-Silva ${ }^{1,2}$ \\ ${ }^{1}$ Laboratório de Resolução da Resposta Inflamatória do Instituto de Ciências Biológicas da Universidade Federal de Minas Gerais, Belo Horizonte, Brasil \\ ${ }^{2}$ Instituto de Ensino e Pesquisa (IEP), Hospital Santa Casa de Belo Horizonte, Belo Horizonte, Minas Gerais, Brasil
}

*Corresponding author: Albená Nunes-Silva, Rua Major Lopes n 738 - apto 601 - Bairro São Pedro - Belo Horizonte - Minas Gerais - Brasil, CEP 30330-050, Tel: 31-9992-3426; E-mail: albenanunes@hotmail.com

Copyright: (c) 2014 Nunes-Silva A, et al. This is an open-access article distributed under the terms of the Creative Commons Attribution License, which permits unrestricted use, distribution, and reproduction in any medium, provided the original author and source are credited.

Received date: April 23, 2014; Accepted date: June 25, 2014; Published date: July 10, 2014

\begin{abstract}
Intense exercise is a physiological stress capable of inducing damage in the exercised muscle group. As a consequence of this exercise-induced micro lesion, there is an increase in the interaction of leukocytes (neutrophils and monocytes/macrophages) with muscle endothelial cells and their transmigration into skeletal muscle tissue. Mechanisms driving this physiological response are not known, but exercise-induced muscle damage may be associated with a local inflammation involving leukocyte accumulation in damaged muscle tissue. Important recent findings show that this infiltration of leukocytes plays an important role in the skeletal muscle remodeling process by activating angiogenesis and hypertrophic pathways inside the skeletal muscle tissue. There are some speculations suggesting that this inflammatory process should be controlled by anti-inflammation drugs in athletes to accelerate the recovery time. Investigations of the literature in this field up to this point do not offer a sufficient body of evidence confirming the need for an anti-inflammatory drug administration to control the exercise-induced inflammation. On the other hand, anti-inflammatory drug administration as a supplemental diet could inhibit important pathways of tissue adaptative responses and block the benefits of exercise to the skeletal muscle tissue.
\end{abstract}

Keywords: Exercise; Inflammation; Anti-inflammatory; Medication

\section{Introduction}

Recently, a variety of studies have demonstrated that exercise induces considerable physiological change in the immune system $[1,2]$. Physical exercise (specifically intense, non-usual and eccentric muscle action) is associated with local skeletal muscle inflammation [3-5]. Following these types of exercise, there are signals of disruptions in the cytoskeleton, fiber disorganization, edema, and a decrease of skeletal muscle function. These typical responses may occur because of the mechanical overload [5].

This type of physical exercise (and consequently, muscle damage) is also associated with an increase in circulating muscle proteins, such as creatine kinase (CK) and myoglobin [5]. Structural abnormalities in the muscle that are also associated with this type of damage include sarcolemmal disruption, distortion of the myofibrillar component, Zline streaming, fragmentation of the sarcoplasmic reticulum, lesions in the plasma membrane, changes in the extracellular myofiber matrix, and swollen mitochondria [5].

There is a growing body of evidence showing that dead cells, or cells undergoing various types of physical or chemical stress, trigger a potent inflammatory response by releasing necrosis-derived products, including genomic and mitochondrial DNA, formyl-peptides, F-actin and reactive oxygen species (ROS) [6]. Once within the interstitium, these molecules contribute to activating resident cells to release chemotactic mediators [6,7], which may act on leukocytes and make them roll, adhere to, and transmigrate through the endothelial wall [8-10]. Inside the skeletal muscle cells, leukocytes produce and release some mediators, as well as vascular endothelial grow factor (VEGF) and cytokine IL-6.
These mediators contribute to the remodeling process of this tissue once the phagocytosis of muscle debris by macrophages modulates the response of muscle to acute injury. Macrophages help to orchestrate the remodeling of vascular and skeletal muscle tissue [11]. During and after intense or repeated skeletal muscle contractile activity, which does not cause cell death but rather causes intense cell stress, there is also a robust increase in the releasing of specific mediators and local inflammatory response.

In this review article, the role of inflammatory process in the skeletal muscle tissue adaptation after exercise, and the recommendation of inflammatory drug administration as a diet supplementation is discussed.

\section{Methods}

The investigation of literature utilized scientific databases such as Pubmed, Bireme and Scielo, by employing the following keys words: exercise, inflammation, anti-inflammatory, and medication. More than 100 articles were located, of which 32 were relevant to this manuscript.

\section{Discussion}

Intense physical exercise represents a metabolic and immunological stress to the human organism. This kind of exercise induces transient changes of leukocyte numbers within circulation that may also reflect hormonal changes (adrenergic and cortisol) and [1-4]. This increase also happens in specific cell numbers, such as neutrophils, monocytes and lymphocytes [12]. Whether persistent changes of leukocyte numbers through repeated intense exercise are able to cause suppression of immunological responses is still an open question. 
Changes in leukocytes counts after exercise appear to be associated with mobilization from the marginal pool and bone morrow [12]. After this mobilization, it is possible to observe a rise in the interaction between leukocytes and endothelial cells in the skeletal muscle tissue; consequently, diapedesis of leukocytes from the blood then stream into skeletal muscle interstitium, characterizing an inflammatory response $[5,13]$.

Altogether, these results show that intense exercise is capable of causing neutrophil-endothelial cell interactions in the microvasculature of exercised muscles and inflammation. A few hours after intense exercise there is a disruption of the fiber alignment and partial destruction of the muscle fibers, as determined by histology studies. In the last decade, it has become clear that acute exercise can induce significant changes in skeletal muscle [3-6]. These changes are characterized by ultra structural loss of muscle architecture, increase of muscle proteins in the bloodstream including creatine kinase $(\mathrm{CK})$ and myoglobin $(\mathrm{Mb})$, a loss of muscular strength and a range of movement and muscle pain [3-5].

Exercise-induced muscle damage may be associated with a local inflammatory response involving leukocyte accumulation in damaged muscle tissue [3,13-15]. Different methods have been used to detect leukocytes accumulation into skeletal muscle tissue, including myeloperoxidase (MPO) biochemical assay, antibody staining procedure (immunohistochemistry or immunofluorescence) and white blood cell (WBC) radionuclide imaging $[14,15]$. Therefore, these results clearly demonstrate that a physiological stimulus (exercise) induces leukocyte influx - a hallmark of inflammation - in the muscle, reinforcing the idea that inflammation is a physiological host response which may have an important role in the regeneration tissue process. It remains elusive whether infiltrating neutrophils contribute to skeletal muscle damage or to skeletal muscle tissue repair $[3,4,14,15]$. There is some evidence that neutrophils induce the production of proinflammatory molecules that may contribute to the remodeling and repair process by activating satellite cells and liberating angiogenic factors [15-17]. The relevance of leukocyte influx into the skeletal muscle tissue is not known yet and clearly deserves further investigation.

It has been suggested, however, that exercise-associated inflammation may be relevant for muscle remodeling and adaptation to the new load of exercise [5]. This remodeling process could occur by increasing in the hypertrophic signaling pathway of the skeletal muscle cell, or by inducing angiogenesis in the tissue [11]. Skeletal muscle is capable of full regeneration, with recovery of its all functions and "up regulation" in its strength and muscle size.

Much of this response is due to the properties of satellite cells, the main adult muscle stem cells, which activate after damage and expand before differentiating and fusing to form new myofibers [17]. Monocytes/macrophages have regularly been observed during muscle regeneration. Specific depletion of circulating monocytes with diphtheria toxin or clodronate-encapsulated liposomes shows a rather severe impairment of muscle regeneration, indicating a critical involvement of these cells in muscle regeneration [18].

The first studies have suggested that macrophages that are present during the first phases of muscle regeneration differ from those that accompany the late phases of the process, and this inflammatory response kinetics was recently confirmed, by using new immunological tools [18].
Quickly after injury, monocytes/macrophages infiltrate the injured muscle area, and then these cells switch their phenotype towards a M2 resolving/wounding phenotype, likely upon phagocytosis of muscle debris [18]. Thus, skeletal muscle regeneration is characterized by a sequence of M1 then M2 macrophages, which has been confirmed during human muscle regeneration, and the phenotype of these populations evolves with time [18]. At the end of muscle regeneration, not only the number of macrophages decrease, but also the phenotype of both changes towards a dampening of all cytokine markers, suggesting a skewing into M2 resolving/silencing macrophages [18].

It is reasonable to conclude that changes in these kinetics greatly alter muscle regeneration. In addition, too-early anti-inflammatory signal releasing, as well as the blocking of later anti-inflammatory signals, could impede muscle regeneration. The pioneer studies on macrophages during muscle regeneration suggested various effects of macrophage subsets on myogenic cell populations. These have been confirmed through a series of in vitro and in vivo analyses [11]. The mechanism which the intense exercise protocol can increase the number of rolling, adherent and transmigrating leukocytes could be explained by the increase in the expression of E-selectin, L-selectin and PECAM by oxidative stress.

One potential function of inflammatory process may be to orchestrate the resolution of the function of the damaged tissue and induce the proper adaptive response to exercise training. Infiltration of neutrophils after exercise may also contribute to the exercise remodeling processes, including angiogenesis and hypertrophic response [11]. The damage of the skeletal muscle induces a complex and coordinated response that involves the interactions of many different cell populations and promotes inflammation, vascular remodeling, and finally, muscle regeneration [11]. Muscle disorders exist in which the irreversible loss of tissue integrity and function is linked to defective neo-angiogenesis, with persistence of tissue necrosis and inflammation [11]. Nunes-Silva et al. using a sophisticated intravital confocal technique, have shown that treadmill exercise induces neutrophil recruitment into muscle tissue in a reactive oxygen species- dependent manner. In this study they employed the progressive fatiguing exercise protocol [19]. The authors found important results, such as the increase in interaction of neutrophils with endothelial cells in quadriceps muscle by intravital microscopy. They also observed clear rolling, adhesion and subsequent transmigration of neutrophils induced by exercise, and their results are consistent with other studies demonstrating leukocyte accumulation in tissue as determined by histology $[1,19,20]$.

When leukocytes infiltration is compromised, endothelial-derived progenitors undergo a significant endothelial to mesenchymal transition, possibly triggered by the activation of morphogenetic protein signaling, collagen accumulation, and the muscle replacement with fibrotic tissue [11]. Taken together, this data suggests that enhanced leukocyte-endothelium interaction, which coordinates leukocytes infiltration to the exercised skeletal muscles, may have relevance in the proper recovery of skeletal muscle tissue after an intense exercise session. Further research considerations include: Is the local skeletal muscle inflammatory process important to the recovery (remodeling) of the tissue in the sports scenario? Is it possible to modulate this response? Should we interfere in this process? What should we do: accelerate, inhibit or respect the natural course time (kinetic inflammation)?

Many authors are questioning these issues within the scientific literature. Even if the results are not clear, Peterson described how 
therapeutic doses of ibuprofen or acetaminophen (anti-inflammatory drug) do not blunt muscle inflammatory cell concentrations 24 hours after a novel bout of eccentric contractions in humans [19]. However, Machida affirms that ibuprofen administration during endurance training cancels running-distance-dependent adaptations of skeletal muscle in mice [20]. The important observation here is that the first study investigated acute response, and the second study observed chronic response of anti-inflammatory administration. Kruger et al. investigated the daily supplementation of proanthocyanidolic oligomer (PCO) before and after experimental skeletal muscle contusion injury, and the results demonstrate that this antiinflammatory blunted neutrophil response in tissue, quicker macrophage infiltration into muscle, and faster recovery due to a left shift in time course of inflammation [21].

The most studied anti-inflammatory drugs are Nonsteroidal AntiInflammatory Drugs (NSAIDs), and Schoenfeld described how a vast number of athletes who consume NSAIDs for exercise-induced muscle damage believe that this habit permits a training session when faced with muscle soreness or injury. The data from this study suggest a negative regulatory effect of NSAIDs on the number of neural cell adhesion molecules (NCAM) in human muscle cells, supporting the view that the cyclo-oxigenase (COX) enzime pathway is important for normal satellite cell activity [22].

There is little reason to believe that the occasional use of NSAIDs or other anti-inflammatory technique will negatively affect muscle growth, but the hypertrophic effects of the chronic use of NSAIDs is less clear. In those who are untrained, it does not appear that regular NSAID use will impede growth in the short term. Given their reported impairment of satellite cell activity, however, longer-term use may well be detrimental, particularly in those who possess greater growth potential.

Future research should seek to clarify inconsistencies between studies, as well as investigating the effects of NSAIDs on muscle hypertrophy in trained subjects and athletes who are known to be frequent consumers of these drugs. Interpretation of these results, in combination with those which may indicate that treatment for inflammation during the training period with ibuprofen, may negatively regulate muscle adaptation. Further investigations at earlier and later time points are needed to conclude whether over-the-counter doses of these drugs are an appropriate treatment for inflammation caused by a novel bout of eccentric contractions.

Given the vital role satellite cells play in skeletal muscle maintenance and regeneration, any factors that interfere with their activity could negate the anabolic effects exerted by exercise, and while the precise mechanism by which the COX pathway acts on satellite cells and the ensuing events in the repair or hypertrophy process remains unclear, there is mounting evidence for a positive regulatory effect on satellite cell activity [17]. Isoprofen administration does not promote any change on tolerance to exercise and does not increase the performance in a single strength training session [23].

Recent statement position shows that healing requires inflammation, that exercised-induced skeletal muscle damage may develop delayed onset muscle soreness (DOMS) [24,25] and that macrophages are important cell for tissue repair and regeneration $[26,27]$. In addition, exercising intensely causes a systemic and local immune response, and this inflammatory process is important to the healing and remodeling to the skeletal muscle cell (muscle fiber). When skeletal muscle tissue and other tissues are damaged, the immune system is activated and there is a local inflammatory process that promotes healing.

The response to both infection and tissue damage looks the same. Inflammatory cells rush to injured tissue to start the healing process. Inside the skeletal muscle interstitium, leukocytes release a hormone as insulin-like growth factor (IGF-1) into the damaged tissues, which helps muscles and other injured parts to heal. The administration of any kind of inflammatory drugs can block this important process to remodeling, and may also inhibit the adaptation of skeletal muscle tissue that permit an overload increase in the exercise training protocol. Therefore, any drugs that reduce inflammation could also delay the remodeling process in the skeletal muscle tissue.

Medication drugs that reduce your immune response could also delay skeletal muscle regeneration. Machida and Takemasa have shown that ibuprofen administration during endurance training cancels running distance dependent adaptations of skeletal muscle in mice [28]. In additon, when NSAID use is combined with exercise, there is some evidence that the inflammatory process in the skeletal muscle and brain may be exacerbated [29].

Mikkelsen et al. investigated the effect of a local NSAID infusion on satellite cells after unaccustomed eccentric exercise in vivo in human skeletal muscle. An NSAID was infused via a microdialysis catheter into the vastus lateralis muscle of one leg (NSAID leg) before, during, and after exercise, with the other leg working as a control (unblocked leg). The number of inflammatory cells (CD68 or CD16 cells) was not significantly increased in either of the legs 8 days after exercise and was unaffected by the NSAID [30]. Non steroidal anti-inflammatory drugs (NSAIDs) have also been used to reduce the pain in athletes and for the population that perform recreational exercise, but results from scientific word appear to show that the relatively mild inflammation does not actually need any alleviation by treatment with NSAIDs [31].

Mackey et al. investigated whether the consumption of NSAIDs could affect satellite cell activity in humans. To investigate this, 14 healthy male endurance athletes volunteered for the study, which involved running $36 \mathrm{~km}$. They were divided into two groups and received either $100 \mathrm{mg}$ indomethacin per day, or a placebo. Muscle biopsies collected before the run and on days 1,3 , and 8 afterward were analyzed for satellite cells by immunohistochemistry with the aid of neural cell adhesion molecule (NCAM) and fetal antigen-1 (FA1) antibodies. Muscle biopsies were also collected from untrained individuals for comparison. When compared with pre-exercise levels, a $27 \%$ increase in the number of NCAM cells was observed on day 8 post-exercise in the placebo group, while levels remained similar at all time points in the NSAID group. No change was seen in the proportion of FA1 cells, although lower levels were found in the muscle of endurance-trained athletes compared with untrained individuals. These results suggest that ingestion of anti-inflammatory drugs attenuates the exercise-induced increase in satellite cell number, supporting the role of the cyclooxygenase pathway in satellite cell activity [32].

Thus, it is becoming clear that healing is delayed by antiinflammatory drugs and almost all pain-relieving medicines, such as non-steroidal anti-inflammatory drugs (like ibuprofen) and immune suppressants that are often used could block the immune response to injury. 


\section{Conclusion}

There is a body of information that describes describes the exerciseinduced inflammation as a physiological response that happens after acute episode of exercise, and chronically induces the adaptation of skeletal muscle tissue after a period of time training. In this sense, antiinflammatory administration as a supplemental diet could inhibit these important adaptative responses and block the benefits of exercise to recreational level and high level of exercise training. Finally, recent literature does not offer a consensus to support diet supplementation of anti-inflammatory drugs to help in the exercise scenario.

\section{References}

1. Pedersen BK, Hoffman-Goetz L (2000) Exercise and the immune system: regulation, integration, and adaptation. Physiol Rev 80: 1055-1081.

2. Nieman DC, Pedersen BK (1999) Exercise and immune function. Recent developments. Sports Med 27: 73-80.

3. Tidball JG (2005) Inflammatory processes in muscle injury and repair. Am J Physiol Regul Integr Comp Physiol 288: R345-353.

4. Peake JM, Suzuki K, Wilson G, Hordern M, Nosaka K, et al. (2005) Exercise-induced muscle damage, plasma cytokines, and markers of neutrophil activation. Med Sci Sports Exerc 37: 737-745.

5. Peake JM, Nosaka K, Suzuki K (2006) Characterization of inflammatory responses to eccentric exercise in humans. Exerc Immunol Rev 11: 64-85.

6. Cheung K, Hume P, Maxwell L (2003) Delayed onset muscle soreness : treatment strategies and performance factors. Sports Med 33: 145-164.

7. Cooper DM, Radom-Aizik S, Schwindt C, Zaldivar F Jr (2007) Dangerous exercise: lessons learned from dysregulated inflammatory responses to physical activity. J Appl Physiol (1985) 103: 700-709.

8. Kubes P, Mehal WZ (2012) Sterile inflammation in the liver Gastroenterology 143: 1158-1172.

9. Chen GY, Nuñez G (2010) Sterile inflammation: sensing and reacting to damage. Nat Rev Immunol 10: 826-837.

10. Ley K, Laudanna C, Cybulsky MI, Nourshargh S (2007) Getting to the site of inflammation: the leukocyte adhesion cascade updated. Nat Rev Immunol 7: 678-689.

11. Zordan P, Rigamonti E, Freudenberg K, Conti V, Azzoni E, et al. (2014) Macrophages commit postnatal endothelium-derived progenitors to angiogenesis and restrict endothelial to mesenchymal transition during muscle regeneration. Cell Death Dis 5: e1031.

12. Kakanis, MW, Peake J, Brenu EW, Simmonds M, Gray B, et al. (2010) The open window of susceptibility to infection after acute exercise in healthy young male elite athletes. Exerc Immunol Rev 16: 119-137.

13. Christer Malm, Pernilla Nyberg, Marianne Engstrom, Bertil Sjodin, Rodica enkei, et al. (2000) Immunological changes in human skeletal muscle and blood after eccentric exercise and multiple biopsies. J Physiol 529: 243-262.

14. Schneider BS, Tiidus PM (2007) Neutrophil infiltration in exerciseinjured skeletal muscle: how do we resolve the controversy? Sports Med 37: 837-856.

15. Smith C, Kruger MJ, Smith RM, Myburgh KH (2008) The inflammatory response to skeletal muscle injury: illuminating complexities. Sports Med 38: 947-969.
16. Butterfield TA, Best TM, Merrick MA (2006) The dual roles of neutrophils and macrophages in inflammation: a critical balance between tissue damage and repair. J Athl Train 41: 457-465.

17. Vierck J, O'Reilly B, Hossner K, Antonio J, Byrne K, et al. (2000) Satellite cell regulation following myotrauma caused by resistance exercise. Cell Biol Int 24: 263-272.

18. Chazaud B (2014) Macrophages: supportive cells for tissue repair and regeneration. Immunobiology 219: 172-178.

19. Nunes-Silva A, Bernardes PTT, Rezende BM, Lopes F, Gomes EC, et al. (2014) Treadmill exercise induces neutrophil recruitment into muscle tissue in a reactive oxygen species-dependent manner. An intravital microscopy study. Plos One 9: e96464.

20. Peterson JM, Trappe TA, Mylona E, White F, Lambert CP, et al. (2003) Ibuprofen and acetaminophen: effect on muscle inflammation after eccentric exercise. Med Sci Sports Exerc 35: 892-896.

21. Machida M, Takemasa $\mathrm{T}$ (2010) Ibuprofen administration during endurance training cancels running-distance-dependent adaptations of skeletal muscle in mice. J Physiol Pharmacol 61: 559-563.

22. Kruger MJ, Myburgh KH, Smith C (2014) Contusion injury with chronic in vivo polyphenol supplementation: leukocyte responses. Med Sci Sports Exerc 46: 225-231.

23. Correa CS, Cadore EL, Baroni BM, Silva ER, Bijoldo JM, et al. (2013) Effects of prophalactic anti-inflammatory non-steroidal ibuprofen on performance in a session of strength training. Rev Bras Med Esporte 19: 116-119.

24. Schoenfeld BJ (2012) The use of nonsteroidal anti-inflammatory drugs for exercise-induced muscle damage: implications for skeletal muscle development. Sports Med 42: 1017-1028.

25. Cheung K, Hume P, Maxwell L (2003) Delayed onset muscle soreness : treatment strategies and performance factors. Sports Med 33: 145-164.

26. Chazaud B (2014) Macrophages: supportive cells for tissue repair and regeneration. Immunobiology 219: 172-178.

27. Saclier M, Cuvellier S, Magnan M, Mounier R, Chazaud B (2013) Monocyte/macrophage interactions with myogenic precursor cells during skeletal muscle regeneration. FEBS J 280: 4118-4130.

28. Machida M, Takemasa $T$ (2010) Ibuprofen administration during endurance training cancels running distance dependent adaptations of skeletal muscle in mice. J Physiol Pharmacol 61: 559-563.

29. Enos RT, Davis JM, McClellan JL, Murphy EA (2013) Indomethacin in combination with exercise leads to muscle and brain inflammation in mice. J Interferon Cytokine Res 33: 446-451.

30. Mikkelsen UR, Langberg H, Helmark IC, Skovgaard D, Andersen LL, et al. (2009) Local NSAID infusion inhibits satellite cell proliferation in human skeletal muscle after eccentric exercise. J Appl Physiol (1985) 107: 1600-1611.

31. Järvinen TA, Järvinen M1, Kalimo H2 (2014) Regeneration of injured skeletal muscle after the injury. Muscles Ligaments Tendons J 3: 337-345.

32. Mackey AL, Kjaer M, Dandanell S, Mikkelsen KH, Holm L, et al. (2007) The influence of anti-inflammatory medication on exercise-induced myogenic precursor cell responses in humans. J Appl Physiol (1985) 103: $425-431$. 